\title{
Heated lidocaine/tetracaine patch for treatment of patellar tendinopathy pain
}

\section{Arnold R Gammaitoni' \\ Henry T Goitz ${ }^{2}$ \\ Stephanie Marsh ${ }^{2}$ \\ Thomas B Marriott ${ }^{3}$ \\ Bradley S Galer'}

'Pain Group, Nuvo Research US, West Chester, PA, USA; ${ }^{2}$ Sports Medicine, Detroit Medical Center, Warren, MI, USA; ${ }^{3}$ Pain Group, Nuvo Research US, Salt Lake City, UT, USA
Correspondence: Arnold R Gammaitoni Pain Group, Nuvo Research US,

I740 Lenape Road, West Chester, PA 19382, USA

Tel +| 484653 |386

Fax + I 6107932125

Email agammaitoni@nuvoresearch.com
This article was published in the following Dove Press journal: Journal of Pain Research

18 July 2013

Number of times this article has been viewed

Introduction: The pain of patellar tendinopathy (PT) may be mediated by neuronal glutamate and sodium channels. Lidocaine and tetracaine block both of these channels. This study tested the self-heated lidocaine-tetracaine patch (HLT patch) in patients with PT confirmed by physical examination to determine if the HLT patch might relieve pain and improve function.

Methods: Thirteen patients with PT pain of $\geq 14$ days' duration and baseline average pain scores $\geq 4$ (on a $0-10$ scale) enrolled in and completed this prospective, single-center pilot study. Patients applied one HLT patch to the affected knee twice daily for 2-4 hours for a total of 14 days. Change in average pain intensity and interference (Victorian Institute of Sport Assessment [VISA]) scores from baseline to day 14 were assessed. No statistical inference testing was performed.

Results: Average pain scores declined from $5.5 \pm 1.3$ (mean \pm standard deviation) at baseline to $3.8 \pm 2.5$ on day 14. Similarly, VISA scores improved from $45.2 \pm 14.4$ at baseline to $54.3 \pm 24.5$ on day 14 . A clinically important reduction in pain score $(\geq 30 \%)$ was demonstrated by $54 \%$ of patients.

Conclusion: The results of this pilot study suggest that topical treatment that targets neuronal sodium and glutamate channels may be useful in the treatment of PT.

Keywords: patellar tendinopathy, patellar tendinosis, heated lidocaine/tetracaine patch, topical analgesic patch, knee pain

\section{Introduction}

For professional and recreational athletes, painful patellar tendinopathy (PT), or "jumper's knee," is a condition that may interfere with performance and can even end an athletic career. ${ }^{1,2}$ While the etiology of PT is unclear, research suggests that tendon overuse without adequate rest causes repeated tendon microtrauma. A failed healing response leads to degeneration of the tendon, characterized by structural, cellular, and biochemical dysregulation; neovascularization; nerve proliferation; and, ultimately, the primary symptom of pain., ${ }^{1,3}$

The pain associated with PT has typically been treated conservatively with nonsteroidal anti-inflammatory drugs (NSAIDs) or cortisone injections, ${ }^{1}$ despite the fact that prostaglandin $\mathrm{E}_{2}$ is not present in PT, and thus, the pain associated with PT is most likely noninflammatory in nature. ${ }^{4}$ Several other hypotheses have been put forward regarding the source of PT pain, including neuropathy as a contributing factor. ${ }^{5}$ Recent research suggests that PT pain may have neurogenic origins, including identification of newly sprouted substance P-positive sensory nerve fibers, ${ }^{6}$ and the expression of the substance P receptor neurokinin-1 (in blood vessels, nerves, and tenocytes), ${ }^{7}$ as 
well as glutamate and its receptor, the N-methyl-D-aspartate (NMDA) receptor. ${ }^{4,8}$ Other mechanosensitive and voltagegated ion channels, such as TREK-1, a tandem-pore-domain potassium channel $\left(2 \mathrm{PK}^{+}\right)$, and voltage-operated calcium channels may also play a role., ${ }^{9,10}$

Glutamate-NMDA receptor signaling is known to have a role in neuropathic pain, ${ }^{11}$ and it thus may represent a novel target for pharmacologic treatment of PT pain. Recently, it was found that local anesthetics, in particular procaine and tetracaine, also block NMDA receptor channels. ${ }^{12-14}$ In addition, local anesthetics, such as lidocaine and tetracaine, are known to relieve pain by inhibiting the depolarization and firing of sensory nerve fibers through sodium channel blockade. ${ }^{15}$ Because of these properties, a local anesthetic containing both lidocaine and tetracaine could pharmacologically target two potential components of PT pain.

One approach to the treatment of PT pain may be a selfheating topical patch that contains lidocaine $70 \mathrm{mg} /$ tetracaine $70 \mathrm{mg}$ (hereafter referred to as the HLT patch). The HLT patch is effective for providing dermal analgesia for venous access and superficial dermatologic procedures. ${ }^{16}$ The use of heat has been shown to enhance drug penetration, ${ }^{17}$ decrease time to onset relative to other topical formulations such as lidocaine/prilocaine cream (EMLA ${ }^{\circledR}$; APP Pharmaceuticals, LLC; Schaumburg, IL, USA), ${ }^{18}$ and improve the efficacy of topical applications of lidocaine and tetracaine. ${ }^{19}$ Application of the HLT patch has been shown to provide analgesic efficacy to an average depth of $8.22 \mathrm{~mm}$ from the skin's surface and a duration of effect lasting at least 2 hours, ${ }^{20}$ suggesting that this patch may have utility for other conditions such as tendinopathies.

The objectives of this prospective, open-label, pilot study were: (1) to evaluate the HLT patch, which targets both sodium channels and NMDA receptors, for its ability to reduce pain associated with PT and to improve function; and (2) to evaluate the tolerability of the HLT patch when administered twice daily for 14 days in the treatment of PT.

\section{Methods}

This was a prospective, open-label, pilot study examining the effectiveness of the topical, self-heating lidocaine/tetracaine patch (Synera ${ }^{\circledR}$; ZARS Pharma, Inc, Salt Lake City, UT, USA) for pain relief and functional improvement in patients with PT. Study approval was given by the local Institutional Review Board and informed consent was obtained from all patients prior to enrollment in the study. Patients were seen by a single practitioner at a single site.
Only patients with isolated PT were eligible for enrollment in the study. The diagnosis was suspected by a history of painful "push off" activities such as jumping, stair climbing, or running that resulted in a painful debilitation. The diagnosis was confirmed by physical examination in which all patients exhibited significant pain with direct palpation to the inferior pole of the patella (proximal site of patellar tendon attachment) while the knee was fully extended, the quadriceps relaxed, and the superior pole of the patella subjected to a posterior force, the latter of which "delivered" the inferior patellar pole to the palpating clinician and reproduced the pain for which the patient sought treatment. Study inclusion required this clinical test to clearly reproduce the pain for which the patient sought treatment. Inclusion criteria for the study were age $\geq 14$ years, PT pain for a minimum 2-week duration, and an average pain intensity score of at least 4 (scale $0-10$, with 0 representing no pain and 10 representing the worst pain imaginable) over the past 24 hours. If the patient had bilateral PT, the knee that was more painful was treated. Exclusion criteria included, but were not limited to, previous surgery in the affected knee, use of class 1 antiarrhythmic drugs, severe hepatic disease, pregnancy, or breastfeeding.

Patient data were collected during two onsite patient visits on days 0 and 14 (or upon early withdrawal) and by telephone on days 7 and 28. On day 0, informed consent was obtained, medical history and concomitant medications were recorded, and a medical exam and assessments were performed. Also on day 0 , patients were instructed on proper use of the HLT patch, advised to avoid strenuous activity during the study, and given study diaries, study drug, and acetaminophen for minor conditions (eg, headache) or as rescue medication for acute episodes of PT pain. Patients who used acetaminophen for PT pain on 2 consecutive days were considered treatment failures. Drug compliance was assessed via a patient diary and by counting unused HLT patches and acetaminophen during the patient visit on day 14 . No additional analgesics or topical treatments for PT were permitted in the study. Patients were instructed to record the following information each evening in their study diaries: average pain score ( $0-10$ point scale) over the last 24 hours, the number of HLT patches used that day, and any use of the provided rescue medication. At day 14, diaries and any unused HLT patches and acetaminophen were collected, assessments were performed, and the skin at the drug application site was evaluated for erythema using a $0-4$ scale: $0=$ no erythema, $1=$ very slight erythema, $2=$ well-defined erythema, $3=$ moderate to severe erythema, and $4=$ severe 
erythema (beet redness) to slight eschar formations (injuries in depth) or other skin reactions. Adverse events (AEs) were recorded during onsite visits and phone interviews.

Patients were instructed to apply one HLT patch directly to the affected knee at home for 2-4 hours twice daily (morning and evening, separated by approximately 8-10 hours). The drug supply for the entire study was dispensed to each patient at the baseline visit (day 0 ). The first HLT patch was to be applied on the evening of day 0 and the final on the morning of day 14 .

To assess pain, patients were asked to rate their average pain intensity during the previous 24 hours on a scale from 0-10. To assess function, patients completed the PT-specific Victorian Institute of Sport Assessment (VISA), ${ }^{21}$ which rates pain interference of physical movements and general sport training on a scale from $0-100(0=$ most pain interference). Patient satisfaction was measured with the Patient Global Assessment of Treatment Satisfaction, which rates satisfaction on a scale from $0-4(0=$ very dissatisfied, $1=$ dissatisfied, $2=$ no preference, $3=$ satisfied, $4=$ very satisfied).

For the purpose of analysis, patients who were considered treatment failures due to acetaminophen use had their baseline pain and VISA scores carried forward. For all analyses, descriptive statistical methods were used. The change from baseline of pain and VISA scores were examined with a Wilcoxon signed-rank test, and $P$-value $<0.05$ was considered statistically significant. All data are presented as the mean \pm standard deviation.

\section{Results}

Thirteen patients enrolled in and completed the study. The date of first patient enrollment was July 2010, and the last patient completed the study in April 2011. Demographic information for the patient population was collected (Table 1 ). The mean age of the patients was $35 \pm 15$ years, and the majority of patients were male $(69 \%)$ and black or African American (54\%). Three patients (23\%) reported having undergone previous physical therapy for PT, and five patients $(31 \%)$ were involved in a physical therapy program for PT during the study. Two patients were deemed treatment failures for using acetaminophen rescue for PT-related pain on $\geq 2$ consecutive days, and one patient did not return the study diary. The mean daily compliance was $86 \% \pm 12 \%$ $(n=12)$.

At baseline, the mean 24-hour average pain score was $5.5 \pm 1.3$, and the mean VISA score was $45.2 \pm 14.4$. After 14 days of HLT patch use, the mean 24-hour pain score
Table I Demographics of patient population at enrollment

\begin{tabular}{ll}
\hline Variables & \\
\hline Sex, n (\%) & $9(69)$ \\
$\quad$ Male & $4(3 \mathrm{I})$ \\
Female & \\
Age & 13 \\
$\mathrm{n}$ & $35(\mathrm{I})$ \\
Mean (SD) & 30 \\
Median & 18,66 \\
$\quad$ Minimum, maximum & \\
Ethnicity, n (\%) & $1(8)$ \\
$\quad$ Hispanic or Latino & $12(92)$ \\
$\quad$ Non-Hispanic, non-Latino & \\
Race, $\mathrm{n}(\%)$ & $7(54)$ \\
$\quad$ Black or African American & $6(46)$ \\
$\quad$ White & \\
\hline
\end{tabular}

Abbreviations: $\mathrm{n}$, number; SD, standard deviation.

improved to $3.8 \pm 2.5(P=0.022)$, and the mean VISA score improved to $54.3 \pm 24.5(P=0.075)$.

Overall, more than half of patients experienced improvement in pain (62\% of patients [8/13]), and $54 \%$ (7/13) were either satisfied or very satisfied with treatment. A continuous responder analysis showed that about 54\% (7/13) of patients had a clinically meaningful improvement in pain defined as a $\geq 30 \%$ reduction (Figure 1).

Posttreatment VISA scores for approximately 50\% of patients ( $6 / 13$ patients) had improved to the point where they were within the VISA score range for patients who do not have tendinopathy (VISA score $\geq 60$ ), ${ }^{21,22}$ with one patient scoring 100 (ie, similar to healthy volunteers).

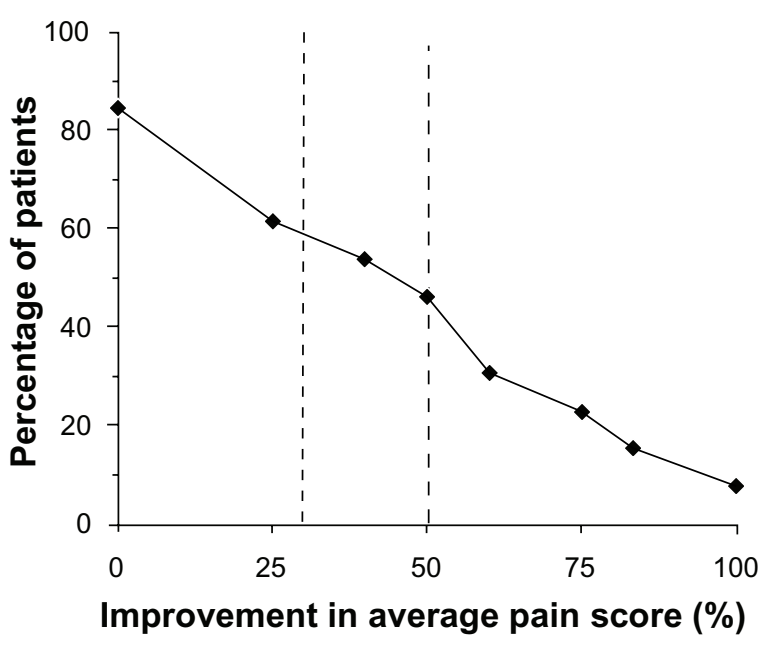

Figure I Patients showed improvement from baseline in 24-hour pain intensity after application of a heated lidocaine/tetracaine patch for 14 days.

Notes: Percent improvement in 24-hour pain intensity ( $0-10$ scale; $0=$ least pain) was calculated using baseline scores for each patient. Fifty-four percent $(54 \%)$ of patients achieved $\mathrm{a} \geq 30 \%$ improvement in pain intensity. 
Treatment-related AEs were mild and included application-site erythema (three patients), application-site dryness (one patient), and headache (one patient).

\section{Discussion}

The pathophysiology of the pain associated with PT is not completely understood but is likely not inflammatory. Recent basic research has suggested that tendon overuse leads to biochemical, structural, and cellular changes to the tendon that may cause PT, ${ }^{1,3}$ and these changes may provide new therapeutic targets for alleviating the pain associated with PT. Recent studies suggest that the pain of PT may be neurogenic in origin, ${ }^{4-8}$ and several neurotransmitters and their receptors may represent potential targets for treatment. Two of these targets include voltage-gated ion channels located on substance P-positive sensory neurons and glutamate signaling via NMDA receptor-1. We therefore conducted this hypothesis-generating, open-label pilot study to explore whether the delivery of local anesthetics via the topical HLT patch could improve patient-reported pain and function by targeting both sodium and NMDA channels. We found that a twice-daily application of the HLT patch for 2 weeks led to a clinically meaningful improvement in pain in $54 \%$ of patients and further improved function by at least $20 \%$ for approximately $50 \%$ of patients. These data appear to support basic research observations that biological targets other than inflammation may play a role in the clinical presentation of pain in the PT patient population.

Until recently, a patient history of pain localized to a tendon would have been referred to as a tendonitis, indicating that the primary cause of pain was inflammation. ${ }^{23}$ What has become evident from basic research studies is that PT is not an inflammatory process. Surgical biopsies have shown that inflammatory cells are absent, ${ }^{4,24}$ and a microdialysis study showed no increased levels of prostaglandin $E_{2}$ in samples from PT patients versus controls. ${ }^{4}$ The absence of an inflammatory mechanism calls into question the routine use of NSAIDs and corticosteroid injections to treat the pain associated with PT, especially since NSAIDs have been shown to be no better than placebo, ${ }^{25}$ and corticosteroid injections do not provide intermediate or long-term benefit. ${ }^{26}$ Because NSAIDs have no inflammatory target in tendinopathy, yet have associated gastrointestinal and cardiovascular risks, they are a suboptimal therapeutic choice for treating the pain associated with PT.

Instead, research has shown that PT and other tendinopathies are more likely a degenerative failed healing response to injury. This tendon degeneration is characterized by collagen loss and disorganization; fibroblast proliferation and fibrosis; increased ground substance; ${ }^{27}$ changes in matrix metalloprotease and metalloprotease-disintegrin expression; ${ }^{28}$ abnormal tenocyte morphology; ${ }^{23}$ hypoxia leading to increased levels of lactate, hypoxia-inducible factor, and vascular endothelial growth factor (VEGF); ;,29 increased neovascularization; ${ }^{30}$ decreased sympathetic and increased sensory nerve growth; ${ }^{6,31}$ as well as changes in other matrix and signaling molecules. ${ }^{28}$ However, it is unclear if these degenerative processes themselves cause pain. More recently, a neurogenic model of PT pain has been proposed. ${ }^{5}$ The results of the present study, in which treatments aimed at interfering with glutamate signaling and blocking of voltagegated ion channels significantly reduced pain due to PT, appear to support the neuropathic pain model of PT.

The only PT therapy that has been repeatedly shown to be successful in controlled studies is eccentric strength training. ${ }^{32,33}$ Pain due to PT was significantly reduced after 12-week and 8-week exercise programs, although strength gains in the latter study were likely prevented by pain during training. ${ }^{34,35}$ While pain during eccentric exercise is allowed in proven rehabilitation programs and under current guidelines, ${ }^{1}$ it is likely to be unpleasant for the patient and may limit the exercise's therapeutic potential. It may be that a combination of eccentric training and analgesic therapy using the topical HLT patch may improve outcomes by facilitating compliance with an eccentric exercise program; however, this hypothesis requires further study.

In our study, we found that repeated application of a single HLT patch applied for 2-4 hours twice a day was generally well tolerated, with no apparent systemic toxicity. A recent pharmacokinetic study examining the use of multiple HLT patches (up to four HLT patches) during a single application showed that lidocaine and tetracaine levels remained far below toxic levels. ${ }^{36}$ For example, Marriott et $\mathrm{al}^{36}$ showed that the plasma $\mathrm{C}_{\text {max }}$ of lidocaine during application of four HLT patches was $18.2 \pm 5.1 \mathrm{ng} / \mathrm{mL}$ for a 2-hour application and $25.7 \pm 5.9 \mathrm{ng} / \mathrm{mL}$ for a 4-hour application, whereas toxic and fatal plasma concentrations of lidocaine are reported to be 1000 -fold higher $(5-31 \mu \mathrm{g} / \mathrm{mL})$. $^{37,38}$ Noninvasive topical therapies, such as the HLT patch, offer the potential advantage of reduced systemic drug exposure and, hence, a reduced risk of systemic AEs. The most common AEs observed in this study were application-site reactions.

This study has substantial limitations. First, the lack of a placebo control and the open-label nature of the trial do not allow one to assess the degree to which placebo response contributed to the findings of the study. Second, the fact 
that patients with only 2 weeks of pain were permitted in the study presents the possibility that patients with a more acute PT pain course were included in the study, and that their pain may have resolved spontaneously. Similarly, no medical imaging procedures were performed to confirm the diagnosis of PT, thus raising the possibility that patients with other pain conditions may have been included. Finally, the small size of the study population precludes rigorous statistical analysis and generalizing these results to a larger PT population.

The goal of this pilot study was to provide initial insights in a clinical setting of a hypothesis generated by the basic research literature: that local therapy targeted to block sodium channels and NMDA receptors could reduce pain in patients with PT. The results of this clinical study suggest that targeting newly identified neurogenic pain mechanisms with the HLT patch may provide clinically important pain relief in patients with PT and improve function. Clearly, larger, adequately controlled trials are required to more fully investigate these effects.

\section{Acknowledgments}

Funding for this prospective case series and to support the preparation of this manuscript was provided by ZARS Pharma, Inc, a wholly owned subsidiary of Nuvo Research Inc. We thank Dr Tara Gupta, Dr Edward Weselcouch, and Diana Talag of PharmaWrite, LLC, for providing medical writing and editorial assistance. This manuscript was prepared according to the International Society for Medical Publication Professionals' Good Publication Practice for Communicating Company-Sponsored Medical Research: the GPP2 Guidelines.

\section{Disclosure}

ARG, TBM, BSG are employees of and have both stock and stock options in Nuvo Research, Inc, which markets the Synera $^{\circledR}$ patch. Dr Goitz has received research payments from ZARS Pharma, Inc, to support the conduct of this study, with assistance from Ms Marsh. The other authors report no other conflicts of interest in this work.

\section{References}

1. Peers KH, Lysens RJ. Patellar tendinopathy in athletes: current diagnostic and therapeutic recommendations. Sports Med. 2005;35(1): $71-87$.

2. Rees JD, Wilson AM, Wolman RL. Current concepts in the management of tendon disorders. Rheumatology (Oxford). 2006;45(5):508-521.

3. Abate M, Silbernagel KG, Siljeholm C, et al. Pathogenesis of tendinopathies: inflammation or degeneration? Arthritis Res Ther. 2009;11(3):235.
4. Alfredson H, Forsgren S, Thorsen K, Lorentzon R. In vivo microdialysis and immunohistochemical analyses of tendon tissue demonstrated high amounts of free glutamate and glutamate NMDAR1 receptors, but no signs of inflammation, in Jumper's knee. J Orthop Res. 2001;19(5):881-886.

5. Webborn AD. Novel approaches to tendinopathy. Disabil Rehabil. 2008;30(20-22):1572-1577.

6. Lian Ø, Dahl J, Ackermann PW, Frihagen F, Engebretsen L, Bahr R. Pronociceptive and antinociceptive neuromediators in patellar tendinopathy. Am J Sports Med. 2006;34(11):1801-1808.

7. Forsgren S, Danielson P, Alfredson H. Vascular NK-1 receptor occurrence in normal and chronic painful Achilles and patellar tendons: studies on chemically unfixed as well as fixed specimens. Regul Pept. 2005;126(3):173-181.

8. Alfredson H, Lorentzon R. Chronic tendon pain: no signs of chemical inflammation but high concentrations of the neurotransmitter glutamate. Implications for treatment? Curr Drug Targets. 2002;3(1): $43-54$.

9. Magra M, Hughes S, El Haj AJ, Maffulli N. VOCCs and TREK-1 ion channel expression in human tenocytes. Am J Physiol Cell Physiol. 2007;292(3):C1053-C1060.

10. Backman LJ, Fong G, Andersson G, Scott A, Danielson P. Substance $\mathrm{P}$ is a mechanoresponsive, autocrine regulator of human tenocyte proliferation. PLoS One. 2011;6(11):e27209.

11. Xu Q, Yaksh TL. A brief comparison of the pathophysiology of inflammatory versus neuropathic pain. Curr Opin Anaesthesiol. 2011; 24(4):400-407.

12. Sugimoto M, Uchida I, Mashimo T. Local anaesthetics have different mechanisms and sites of action at the recombinant N-methyl-D-aspartate (NMDA) receptors. Br J Pharmacol. 2003; 138(5):876-882.

13. Furutani K, Ikoma M, Ishii H, Baba H, Kohno T. Bupivacaine inhibits glutamatergic transmission in spinal dorsal horn neurons. Anesthesiology. 2010;112(1):138-143.

14. Nishizawa N, Shirasaki T, Nakao S, Matsuda H, Shingu K. The inhibition of the N-methyl-D-aspartate receptor channel by local anesthetics in mouse CA1 pyramidal neurons. Anesth Analg. 2002; 94(2):325-330.

15. Ritchie JM, Greengard P. On the mode of action of local anesthetics. Annu Rev Pharmacol. 1966;6:405-430.

16. Curry SE, Finkel JC. Use of the Synera patch for local anesthesia before vascular access procedures: a randomized, double-blind, placebocontrolled study. Pain Med. 2007;8(6):497-502.

17. Tadicherla S, Berman B. Percutaneous dermal drug delivery for local pain control. Ther Clin Risk Manag. 2006;2(1):99-113.

18. Soltesz S, Dittrich K, Teschendorf P, Fuss I, Molter G. [Topical anesthesia before vascular access in children. Comparison of a warmthproducing lidocaine-tetracaine patch with a lidocaine-prilocaine patch.] Anaesthesist. 2010;59(6):519-523. German [with English abstract].

19. Masud S, Wasnich RD, Ruckle JL, et al. Contribution of a heating element to topical anesthesia patch efficacy prior to vascular access: results from two randomized, double-blind studies. J Pain Symptom Manage. 2010;40(4):510-519.

20. Wallace MS, Kopecky EA, Ma T, Brophy F, Campbell JC. Evaluation of the depth and duration of anesthesia from heated lidocaine/tetracaine (Synera) patches compared with placebo patches applied to healthy adult volunteers. Reg Anesth Pain Med. 2010;35(6): 507-513.

21. Visentini PJ, Khan KM, Cook JL, Kiss ZS, Harcourt PR, Wark JD. The VISA score: an index of severity of symptoms in patients with jumper's knee (patellar tendinosis). Victorian Institute of Sport Tendon Study Group. J Sci Med Sport. 1998;1(1):22-28.

22. Frohm A. Patellar Tendinopathy: On Evaluation Methods and Rehabilitation Techniques. Stockholm, Sweden: Karolinska University Press; 2006.

23. Khan K, Cook J. The painful nonruptured tendon: clinical aspects. Clin Sports Med. 2003;22(4):711-725. 
24. Khan KM, Bonar F, Desmond PM, et al. Patellar tendinosis (jumper's knee): findings at histopathologic examination, US, and MR imaging. Victorian Institute of Sport Tendon Study Group. Radiology. 1996; 200(3):821-827.

25. Aström M, Westlin N. No effect of piroxicam on achilles tendinopathy. A randomized study of 70 patients. Acta Orthop Scand. 1992;63(6): 631-634.

26. Coombes BK, Bisset L, Vicenzino B. Efficacy and safety of corticosteroid injections and other injections for management of tendinopathy: a systematic review of randomised controlled trials. Lancet. 2010;376(9754):1751-1767.

27. Khan KM, Cook JL. Overuse tendon injuries: where does the pain come from? Sports Med Arthrosc. 2000;8(1):17-31.

28. Riley G. Tendinopathy - from basic science to treatment. Nat Clin Pract Rheumatol. 2008;4(2):82-89.

29. Scott A, Lian Ø, Bahr R, Hart DA, Duronio V. VEGF expression in patellar tendinopathy: a preliminary study. Clin Orthop Relat Res. 2008; 466(7):1598-1604.

30. Aström M, Rausing A. Chronic achilles tendinopathy. A survey of surgical and histopathologic findings. Clin Orthop Relat Res. 1995; (316):151-164.

31. Danielson P, Alfredson H, Forsgren S. Studies on the importance of sympathetic innervation, adrenergic receptors, and a possible local catecholamine production in the development of patellar tendinopathy (tendinosis) in man. Microsc Res Tech. 2007;70(4):310-324.
32. Larsson ME, Käll I, Nilsson-Helander K. Treatment of patellar tendinopathy - a systematic review of randomized controlled trials. Knee Surg Sports Traumatol Arthrosc. 2012;20(8):1632-1646.

33. Cook JL, Khan KM. What is the most appropriate treatment for patellar tendinopathy? Br J Sports Med. 2001;35(5):291-294.

34. Jensen K, Di Fabio RP. Evaluation of eccentric exercise in treatment of patellar tendinitis. Phys Ther. 1989;69(3):211-216.

35. Cannell LJ, Taunton JE, Clement DB, Smith C, Khan KM. A randomised clinical trial of the efficacy of drop squats or leg extension/leg curl exercises to treat clinically diagnosed jumper's knee in athletes: pilot study. Br J Sports Med. 2001;35(1):60-64.

36. Marriott TB, Charney MR, Stanworth S. Effects of application durations and heat on the pharmacokinetic properties of drug delivered by a lidocaine/tetracaine patch: a randomized, open-label, controlled study in healthy volunteers. Clin Ther. 2012;34(10):2174-2183.

37. Mehra P, Caiazzo A, Maloney P. Lidocaine toxicity. Anesth Prog. 1998; 45(1):38-41.

38. Centini F, Fiore C, Riezzo I, Rossi G, Fineschi V. Suicide due to oral ingestion of lidocaine: a case report and review of the literature. Forensic Sci Int. 2007;171(1):57-62.
Journal of Pain Research

\section{Publish your work in this journal}

The Journal of Pain Research is an international, peer-reviewed, open access, online journal that welcomes laboratory and clinical findings in the fields of pain research and the prevention and management of pain. Original research, reviews, symposium reports, hypothesis formation and commentaries are all considered for publication.

\section{Dovepress}

The manuscript management system is completely online and includes a very quick and fair peer-review system, which is all easy to use. Visit http://www.dovepress.com/testimonials.php to read real quotes from published authors. 